\title{
COMPRESSÃO DA VEIA ILIACA POR DILATAÇÃO DA BEXIGA
}

\section{Vinicius Freitas Pedron'; João Victor Guimarães ${ }^{3}$, Cleoni Pedron², Vinicius Menegola ${ }^{4}$}

\section{RESUMO}

O edema unilateral do membro inferior na maioria dos casos leva a suspeita clinica de trombose venosa profunda (TVP). Algumas patologias não frequentes podem causar edema unilateral mimetizando um TVP. A ocorrencia de distenção vesical com obstrução das veias iliacas é um fenomeno raro de ser descrito. $O$ objetivo do trabalho é relatar a compressão da veia iliaca por bexigoma que pode ser a causa de edema unilateral e ser confundido com uma TVP. Os métodos utilizados para a elucidação do diagnóstico e da terapia, foram a história clínica, o exame fisico, o eco Doppler venoso colorido e a tomografia computadorizada venosa. Este trabalho descreve três pacientes com edema da extremidade inferior causado pela compressão da bexiga nas veias iliacas. A compressão da veia ilíaca pela bexiga urinária é uma causa rara de edema unilateral, mas deve ser incluídas no diagnóstico diferencial dessa patologia.

Palavras-chave: Trombose venosa profunda, edema dos membros inferiores e compressão venosa.

Eixo Temático: Área de Ciências da Saúde.

\section{INTRODUÇÃO}

A compressão das veias ilíacas pela bexiga urinária distendida não é frequentemente relatada na literatura inglesa atualmente disponível. Isso afeta predominantemente homens, que são vulneráveis ao prostatismo (LIN et al., 2014). O edema unilateral do membro inferior está associado comumente a hipótese

\footnotetext{
1 Vinicius Freitas Pedron - Universidade Franciscana (UFN), pedron.vinicius@hotmail.com.

2 Cleoni Pedron - Médico Cirurgião Endovascular, cpedron@uol.com.br.

3 João Victor Guimarães Almeida - Médico Clinico Geral, jvictonis@gmail.com.

${ }^{4}$ Vinicius Matos Menegola - Professor Dr. da Universidade Franciscana (UFN), Médico Cirurgião Vascular, vini_kam@hotmail.com.
} 
diagnóstica de trombose venosa profunda (TVP). Porém algumas outras patologias podem causar edema unilateral sejam elas vasculares ou não vasculares e mimetizar TVP, como linfedema e compressões venosas extrínsecas.

As causas mais comuns de edema não trombótico são as compressões venosas extrínsecas do setor ilíaco, como a Sindrome de May-Thurner. Assim como tumores da região pélvica especialmente miomas e adenocarcinomas de útero. Também aneurismas das artérias ilíacas podem causar compressão venosa. Mais raramente outras estruturas anatômicas pélvicas podem comprimir as veias ilíacas e simular ou causar TVP unilateral. Entre elas está a distensão da bexiga vesical. Que foi relatado a primeira vez por Carlsson e Garsten em 1960. Nós descrevemos a apresentação de três casos de edema unilateral devido a compressão venosa ilíaca extrínseca causada pela distensão da bexiga urinária. Em dois casos não ocorreram TVP e um caso o paciente apresentou quadro de TVP associada a compressão. $O$ objetivo do trabalho é demonstrar outras causas de edema unilateral do membro inferior que não a TVP. A compressão da veia iliaca por bexigoma que pode ser a causa de edema unilateral e ser confundido com uma TVP.

\section{METODOLOGIA}

Todos os pacientes estavam internados no Hospital de Caridade Dr. Astrogildo de Azevedo na cidade de Santa Maria - RS, no serviço de cirurgia vascular e endovascular. O período de coleta de dados foi de 10 anos, de 2010 a 2020.

Este trabalho consta um relato de experiência do serviço, de três pacientes diagnosticados com compressão da veia ilíaca pela dilatação da bexiga urinária. Casos de compressão ilíaca por bexigoma são raramente relatados na literatura.

O paciente I é do sexo feminino, com 71 anos que foi referenciado para o nosso serviço com quadro de edema unilateral a esquerda. Sua história médica pregressa era de ressecção cirúrgica de tumor cerebral (Gliobastoma - Grau IV) no hemisfério direito. Apresentava sequela neurológica de hemiparesia a esquerda.

O paciente II é do sexo masculino de 38 anos com quadro de edema no membro inferior direito (MID). Histórico médico pregresso com diagnóstico de 
esquizofrenia em uso de biperideno $2 \mathrm{mg} / \mathrm{dia}$, cloridrato de sertralina $100 \mathrm{mg} / \mathrm{dia}$ e topiramato $100 \mathrm{mg} / \mathrm{dia}$.

O paciente III é do sexo masculino com 61 anos com história de neoplasia de próstata metastática (osséa e menigea) já submetido a prostatectomia em 2006. Sendo tratado com quimioterapia. Apresentou quadro de edema no mie e distensão abdominal.

A raridade de relatos na literatura médica está atrelado a baixa suspeita clínica de edema causado por compressão da veia ilíaca pela distensão da bexiga. Portanto, o trabalho em questão é de extrema importância.

\section{RESULTADOS E DISCUSSÕES}

\subsection{Resultados}

\subsubsection{Primeiro Paciente:}

Paciente feminina com 71 anos que foi referenciado para o nosso serviço com quadro de edema unilateral a esquerda. Sua história médica pregressa era de ressecção cirúrgica de tumor cerebral (Gliobastoma - Grau IV) no hemisfério direito. Apresentava sequela neurológica de hemiparesia a esquerda.

Um eco Doppler colorido foi realizado que não demonstrou TVP mas presença de compressão venosa proximal.

Uma ultrassonografia abdominal demonstrou distensão importante da bexiga urinária causando compressão da veia ilíaca externa esquerda (figura 1a e 1b). Uma sondagem vesical foi feita com drenagem de 1.4 litros de urina clara com resolução gradual do edema, que resolveu completamente em 3 dias. $\mathrm{O}$ estudo urodinâmico demonstrou presença de bexiga neurogênica. Foi realizada cistostomia permanente. 

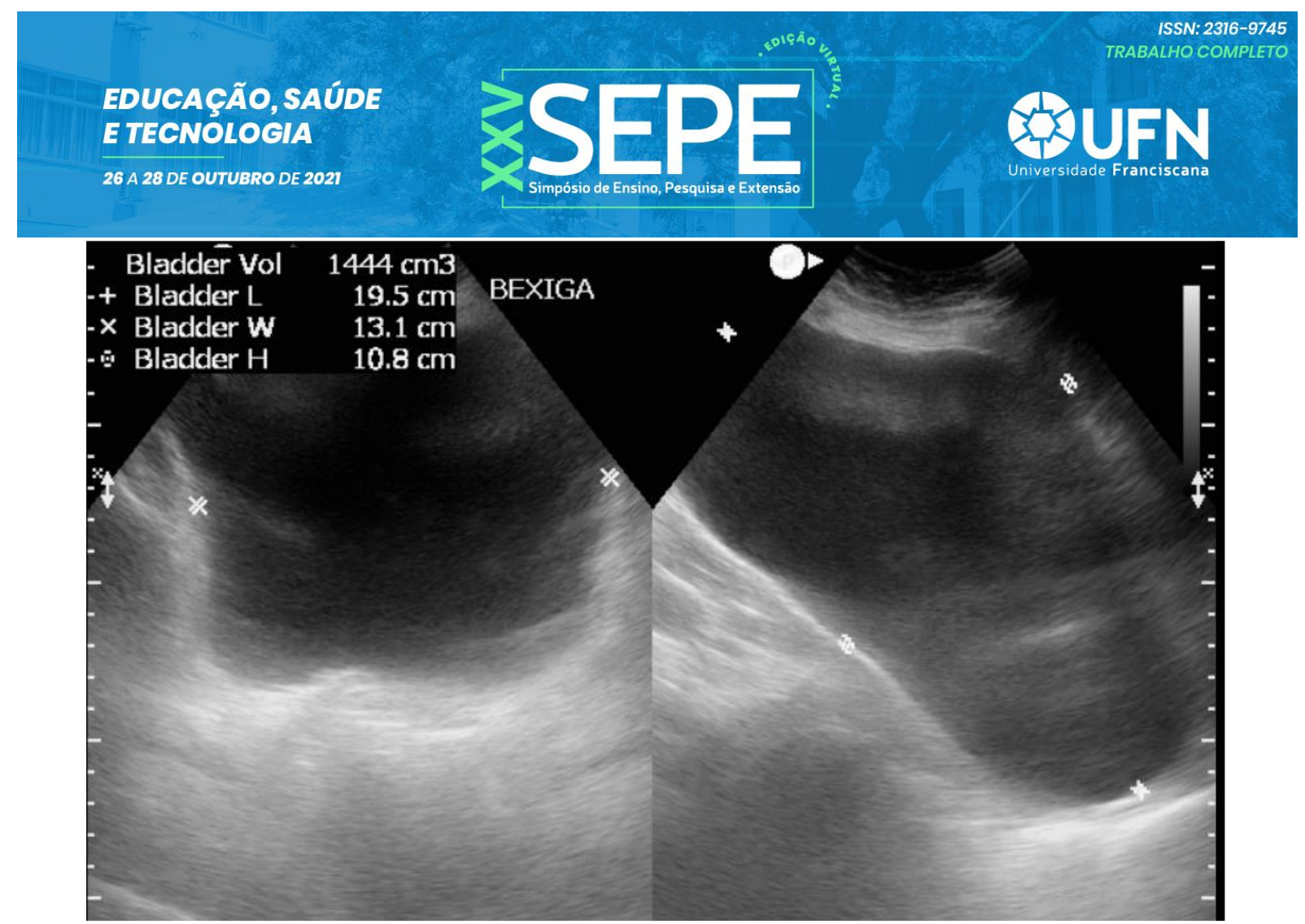

Figura 1a: Ultrassonografia abdominal com distenção da bexiga urinária, volume de $144 \mathrm{~cm}^{3}$.

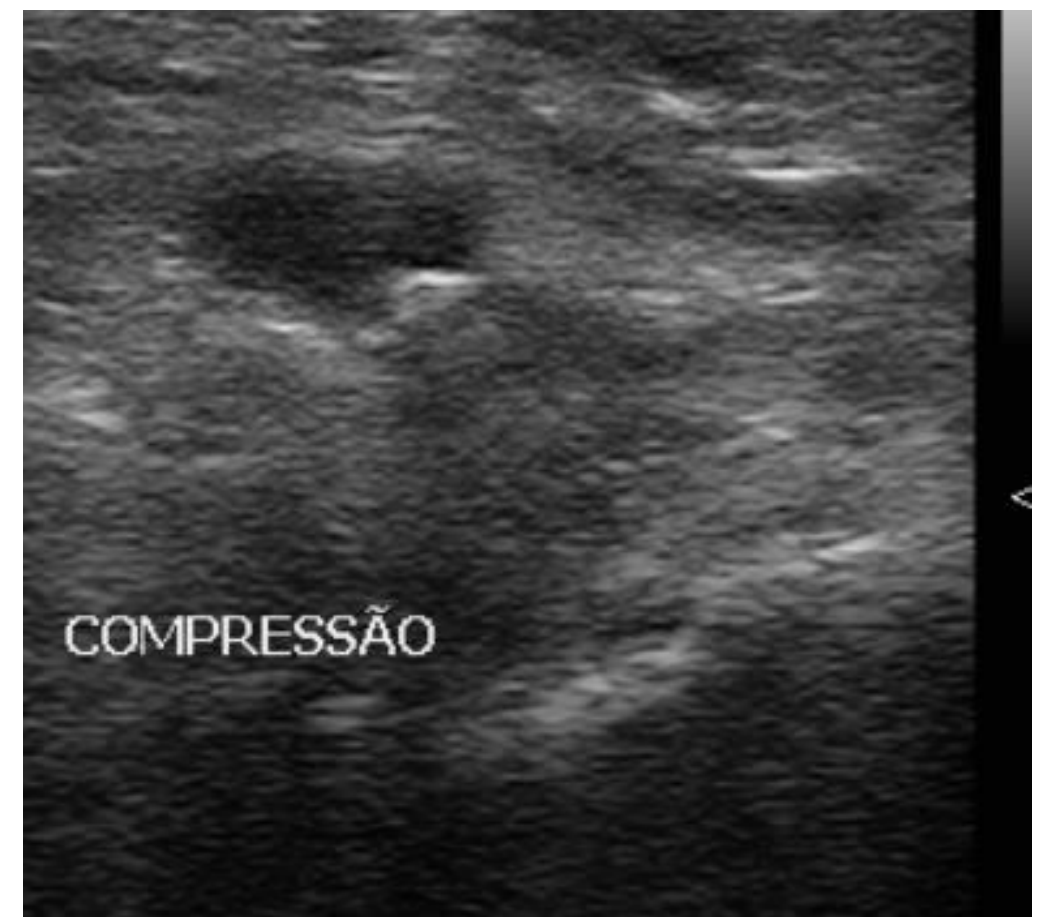

Figura 1b: Compressão da veia iliaca externa pela bexiga urinária distendida. 


\subsubsection{Segundo Paciente:}

Paciente masculino de 38 anos com quadro de edema no membro inferior direito (MID). Histórico médico pregresso com diagnóstico de esquizofrenia em uso de biperideno $2 \mathrm{mg} / \mathrm{dia}$, cloridrato de sertralina $100 \mathrm{mg} / \mathrm{dia}$ e topiramato $100 \mathrm{mg} / \mathrm{dia}$. O eco Doppler colorido venoso evidenciou TVP iliofemoral do MID, de aspecto recente com envolvimento das veias ilíaca externa, femoral comum e superficial direita. Foi realizada trombolise cateter direcionada intravenosa local com punção ecoguiada da veia poplítea e colocação de cateter multiperfurado Fountain (Medrad) no setor ocluído durante 18 horas com dose de $1 \mathrm{mg} / \mathrm{h}$ de RtPA. O controle de flebográfico pós trombolise, com lise completa do trombo e imagem de estenose na veia ilíaca externa direita associada a distenção da bexiga (figura 2a). Foi realizada sondagem vesical de demora durante o procedimento com drenagem de $2.500 \mathrm{ml}$ de urina e repetida a flebografia, com desaparecimento da imagem de compressão externa e normalização do fluxo venoso (figura 2b). Posteriormente, no estudo urodinâmico foi diagnosticado atonia vesical. Provavelmente devido ao uso de biperideno. A pesquisa de trombofilia foi negativa. O medicamento foi suspenso. Um segundo estudo urodinâmico, com melhora do tônus vesical. O paciente evoluiu satisfatoriamente, sem edema e em uso de AVK. O eco Doppler de controle não demostrou sequelas de TVP, assim como fluxo venoso adequado, sem obstrução proximal. 


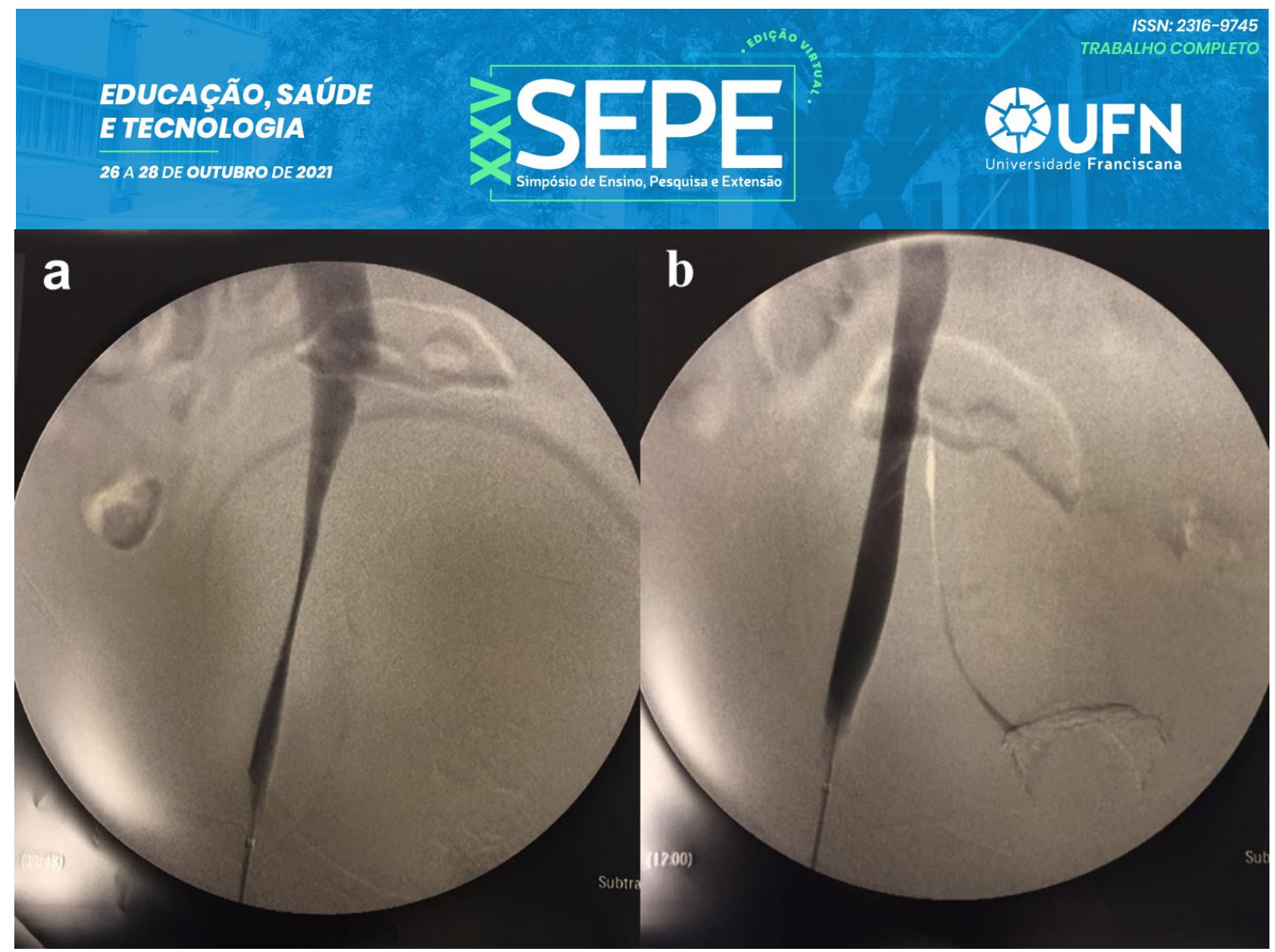

Figura 2: (a) - Flebografia pós-trobolise com ausencia de trombo e identificado compressão pela bexiga urinária; (b) - Controle angiográfico imediato pós sondagem visical de demora com drenagem de $2500 \mathrm{~mL}$ de urina.

\subsubsection{Terceiro Paciente:}

Paciente masculino com 61 anos com história de neoplasia de próstata metastática (osséa e menigea) já submetido a prostatectomia em 2006. Sendo tratado com quimioterapia. Apresentou quadro de edema no mie e distensão abdominal. Foi realizado eco Doppler colorido venoso do mie com ausência de TVP, porém fluxo venoso não fásico com a respiração, sugestivo de compressão proximal. A tomografia de abdome revelou grande distensão da bexiga com efeito compressivo sobre a veia ilíaca comum esquerda e ausência de trombos no setor iliocaval e uronefrose bilateral. A seguir, foi realizada sondagem vesical de demora com drenagem de $2150 \mathrm{ml}$ de urina e realizada uma flebotomografia das veias ilíacas e cava com ausência de distensão da bexiga e de compressão da veia ilíaca com opacificação normal das veias ilíacas e cava inferior pelo meio de contraste e ausência de trombos nos setor estudado (figura 3 a e b). Clinicamente houve resolução do edema no MIE. 

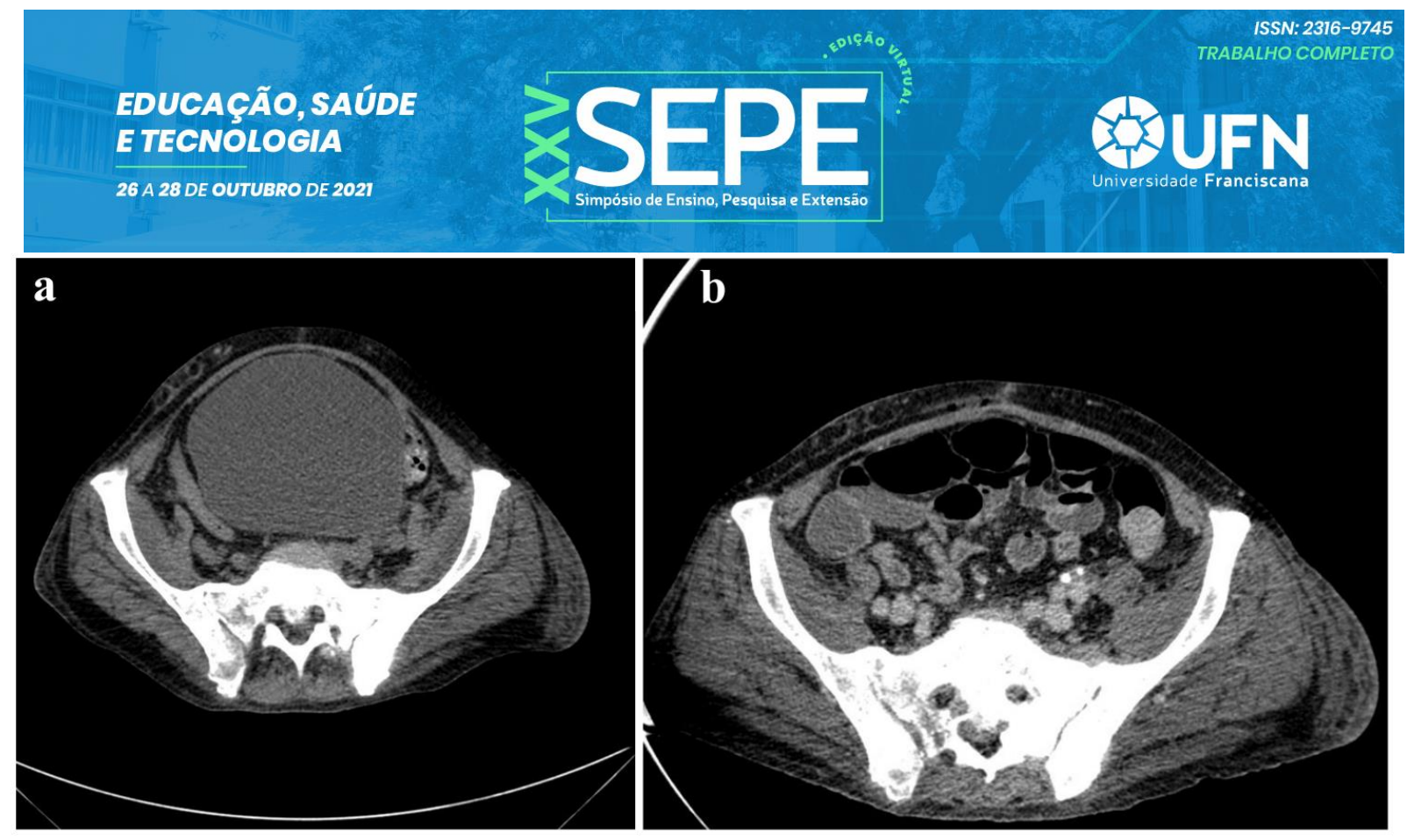

Figura 3: (a) - Tomografia demonstrando grande distenção da bexiga urinária e compressão da veia iliaca esquerda; (b) - Tomografia de abdomem pós sondagem visical de demora.

\subsection{Discussão}

O edema unilateral é na maioria das vezes causado por TVP, mas outras condições podem causar edema como celulite, artrite, fratura, linfedema, hematoma, torn gastrocnemius muscle, Baker's cyst e outros (GORMAN; DAVIS; DONNELLY, 2000; ROSSI et al., 2007). Também outras patologias ou complicações podem causar edema unilateral e TVP por compressão das veias ilíacas como os aneurismas da aorta abdominal e das artérias ilíacas (ROSENTHAL et al., 1998), compressão da veia ilíaca externa por reservatório de implante peniano (ROSENTHAL et al., 1998), tumores pélvicos malignos e benignos (FRCSC; FRCSC; GIBBONS, 2002).

Uma outra forma de compressão venosa das ilíacas é por distensão da bexiga urinária, que foi descrita pela primeira vez em 1960. Essa publicação foi de um recém nascido com quadro de aumento de volume do abdome, aparecimento de hérnia inguinal direita e edema do mie com membro cianótico, pálido e frio. O exame físico demonstrou uma massa abdominal. Uma sonda uretral foi passada com drenagem de $120 \mathrm{ml}$ de urina clara e a massa abdominal não foi mais encontrada. A urografia demonstrou dilatação da bexiga urinária e identificada presença de válvula na parte posterior da uretra. Que foi ressecada. Duas semanas após o procedimento o membro estava completamente normal (CARLSSON; GARSTEN, 1960). 
Em 2012 foi publicado um relato de caso de edema no MIE devido a compressão venosa por distensão da bexiga urinária devido a bexiga neurogênica após uma histerectomia radial (LIN et al., 2014).

Kopesky et al descreveram dois casos de edema bilateral devido a compressão das veias ilíacas pela bexiga urinária devido a hiperplasia benigna de próstata que foi tratada com ressecção transuretral com desaparecimento dos sintomas vasculares (KOPESKY; SCHWARTZ; SILVER, 1988).

Também Palma et al. descreveram um caso de um paciente que apresentou edema no membro inferior esquerdo após a segunda cirurgia para enxerto de pele no mie após acidente automobilístico. $\mathrm{O}$ acidente aconteceu seis meses antes sem lesão espinal, pélvica ou uretral. O paciente apresentou edema no MIE pós o procedimento cirúrgico, após a retirada da sonda vesical e distensão da bexiga urinária. A sondagem vesical foi realizada com drenagem de $4.100 \mathrm{ml}$ com melhora do edema em alguns dias. Foi realizada uma ressecção transuretral da próstata com resolução do edema. A biópsia demonstrou hiperplasia benigna de próstata sem carcinoma. Nessa publicação de 1995 foi realizada um revisão bibliográfica que encontrou 15 casos de edema causado por compressão venosa devido a distensão da bexiga. A grande maioria dos casos era por hiperplasia benigna da próstata. Outras por bexiga neurogênica e um caso por estenose de uretra (PALMA; 1995).

Outra publicação em 1999, por Ducharme et al, de um paciente idoso com demência que apresentou edema no mid devido a compressão da veia ilíaca por uma distensão de bexiga devido a hiperplasia benigna de próstata, que foi tratada com ressecção transuretral (DUCHARME et al., 1999).

Vaidyanathan et al publicou um caso de um homem com paraplegia devido a lesão ao nível de T-5 e bexiga neurogênica e compressão da veia ilíaca esquerda (VAIDYANATHAN et al., 2001).

A paciente 1 permanece assintomática na parte vascular porém ainda com sequelas da lesão neurológica e permanece com a cistostomia. O paciente 2 continua o acompanhamento clínico em uso de de Rivaroxabana ${ }^{\circledR}$ sem sequelas venosas. 0 paciente 3 teve piora do quadro clínico devido a neoplasia de próstata e faleceu cerca de 3 meses após. 


\section{CONCLUSÃO}

Em resumo, a compressão da veia ilíaca pela bexiga urinária é uma causa rara de edema unilateral, mas deve ser incluídas no diagnóstico diferencial dessa patologia. Especialmente nos pacientes com neoplasia do trato urinário e lesões neurológicas que podem afetar a adequada fisiologia da bexiga urinária.

\section{REFERÊNCIAS}

CARLSSON, E. \& GARSTEN, P. Compression of the Common lliac Vessels by Dilatation of the Bladder. Acta Radiologica, n. 53, p. 449-453, 1960.

DUCHARME, C. S. E. et al. Unilateral iliac vein occlusion, caused by bladder enlargement, simulating deep venous thrombosis. p. 724-726, 1999.

GORMAN, W. P.; DAVIS, K. R.; DONNELLY, R. Swollen lower limb - 1: General assessment and deep vein thrombosis. BMJ, v. 320, n. 5, p. 1453-1456, 2000.

JUSTA DG, BIANCO FJ, OGLE A \& DHABUWALA C. Deep Venous Thrombosis Due to Compression of External lliac Vein by the Penile Prosthesis Reservoir. Urology, $\mathrm{n}$. 61, p. 462-463, 2003.

KOPESKY K,SCHWARTZ R \& SILVER D. Lower extremity edema from bladder compression of the iliac veins. Jornal Vascular Surgery, v. 7, p. 778-780, 1988.

LIN, C. et al. Compression on iliac vein from distended urinary bladder. Journal of the Formosan Medical Association, v. 113, n. 9, p. 664-665, 2014.

Palma L, Peterson MC\& Ingebretsen R. Iliac Vein Compression Syndrome From Urinary Bladder Distension Due to Prostatism. Southern Medical Journal, v. 88, n. 9, p. 959-960, 1995.

ROSENGARTEN, A. M.; WONG, J.; GIBBONS, S. Endometriosis causing cyclic compression of the right external iliac vein with cyclic edema of the right leg and thigh. Journal of Obstetrics and Gynaecology Canada, v. 24, n. 1, p. 33-35, 2002.

Rosenthal $\mathrm{D}$, Matsuura $\mathrm{JH}$, Jerius $\mathrm{H}$ \& Clark MD. lliofemoral Venous Thrombosis Caused by Compression of an Internal Aneurysm: a Minimally Invasive Treatment. Journal Endovascular Surgery, v. 5, p. 142-145, 1998.

ROSSI, F. H. et al. Extrinsic compression of the external iliac vein and lower limb 
EDUCAÇÃO, SAÚDE

ETECNOLLOGIA

26 A 28 DE OUTUBRO DE 2021

\section{QUFN}

edema caused by hip joint synovial cyst. 2007.

VAIDYANATHAN, S. et al. Original Article Occlusion of left common iliac vein by a distended urinary bladder in a male with paraplegia due to spinal cord injury. Spinal Cord, p. 394-398, 2001. 